\title{
INFANCIAS INDÍGENAS. LOS CENTROS DE ATENCIÓN A LA NIÑEZ EN CHIAPAS Y EL RETO DE LA EDUCACIÓN INTERCULTURAL FRENTE A LA DIVERSIDAD
}

\author{
Indigenous Childhoods. Care Centers for Children in Chiapas and the Challenge of Intercultural \\ Education for Diverse Populations
}

\author{
Kathia Núñez-Patiño \\ Claudia Molinari-Medina \\ Cecilia Alba-Villalobos
}

\begin{abstract}
Resumen: Las transformaciones económicas que han impactado en las comunidades indígenas en Chiapas desde los últimos años del siglo XX han acelerado procesos de migración hacia ciudades como Tuxtla, Tapachula o San Cristóbal de Las Casas. En esta última localidad hay una creciente presencia de niños y niñas indígenas en espacios públicos que realizan actividades económicas en diversidad de condiciones. En este artículo se identifican modelos de atención a la población indígena infantil que utilizan las organizaciones civiles y dependencias públicas, conel fin de conocer las concepciones de infancia que influyen en los programas de estas instituciones, su eficacia y su pertinencia cultural. Exponemos las características de los centros que ofrecen atención a la infancia, cómo se asume el contexto intercultural y los retos que enfrenta la educación formal.
\end{abstract}

Palabras clave: infancia, población indígena, participación social, educación intercultural.

Abstract: The economic changes that have impacted indigenous communities in Chiapas, since the late twentieth century have accelerated migration to cities like Tuxtla, Tapachula, and San Cristobal de Las Casas. In the latter town, evidence shows an increase in the presence of indigenous children in public places with commercial and tourist areas, in various conditions. This article identifies different models of childcare for indigenous children, found in those operating civil organizations and public agencies, in order to learn the concepts of childhood behind these institutions. We analyze their effectiveness and cultural relevance, and we present the way in which these centers address interculturalism in the provision of childcare and the challenges facing formal education in order to respond to the diversity of indigenous children.

Keywords: childhood, indigenous peoples, social participation, intercultural education.

Kathia Núñez Patiño, candidata a doctora por el Instituto de Investigaciones en Educación de la Universidad Veracruzana. Profesora de tiempo completo en la Facultad de Ciencias Sociales de la Universidad Autónoma de Chiapas, México. Temas de especialización: infancia, educación autónoma, cultura e identidad. Correo electrónico: ktyeel@hotmail.com.

Claudia Molinari Medina, doctora por la Universidad de la Sorbona, París, Francia. Profesora de tiempo completo en la Facultad de Ciencias Sociales de la Universidad Autónoma de Chiapas, México. Temas de especialización: religión, rebeliones de los pueblos étnicos, historia de México del S. XIX. Correo electrónico:clmolinari@hotmail.com.

Cecilia Alba Villalobos, candidata a doctora en Estudios Regionales por la Universidad Autónoma de Chiapas, México. Profesora de tiempo completo en la Facultad de Ciencias Sociales de la Universidad Autónoma de Chiapas, México. Temas de especialización: antropología y educación; Antropología en Chiapas. Correo electrónico: cecilialba03@hotmail.com.
Enviado a dictamen: 21 de mayo de 2015.

Aprobación: 30 de septiembre de 2015.

Revisiones: 1. 


\section{Introducción}

L a mayoría de las políticas dirigidas a la infancia están fuertemente influidas por imágenes dominantes, como los estándares anglosajones y europeos, que provienen principalmente de la psicología del desarrollo y homogeneízan a la población infantil, lo que va en contra del reconocimiento y, por tanto, de la aceptación de la diversidad de infancias, además de asumir a los niños y niñas como personas en formación, sin considerarlos sujetos activos y con voz propia.

En este sentido, desde nuevos enfoques de la sociología, la antropología social y la psicología sociocultural, se hacen críticas a las visiones que caracterizan de una misma manera a la infancia y, desde investigaciones recientes, se señala la importancia de los contextos culturales en el desarrollo de los niños, destacando su papel agentivo, en tanto sujetos sociales, $y$ analizando las formas en que participan dentro de sus entornos (James, Jenks y Prout, 1998; Hirschfeld, 2002; De León, 2010; Gaskin, 2010; Corona y Linares, 2007 y Rogoff, 1993).

Algunos de los señalamientos a la visión de la psicología del desarrollo que se desprenden de los estudios actuales de la infancia refieren que esta concepción se ha construido bajo una jerarquía basada en la edad, desde los intereses de la economía adulta, porque regulariza e institucionaliza las relaciones entre adultos y niños, estableciendo las características y diferencias de la infancia bajo la lógica de esta oposición (James, Jenks y Prout, 1998). Por ende, sólo se da importancia al producto de la socialización, en la que se introducen los valores y normas sociales, así como a las instituciones encargadas del proceso, como la familia y la escuela, lo que invisibiliza o niega la participación infantil en sus contextos.

La crítica a este enfoque adultocéntrico genera que el concepto de infancia se relativice, al considerar este periodo de la vida como una condición social delimitada por una construcción histórica, culturalmente diferenciada y caracterizada por las relaciones de poder en las que se desenvuelven las niñas y los niños (Gaitán, 2006). El nuevo enfoque prepara el terreno para un análisis en términos de su contexto social y abandona el concepto de la reducción naturalista, que pretendía homogeneizar las infancias y legitimar el poder sobre ellas porque, al asociar la infancia con una etapa del "desarrollo natural" más que con la cultura, se construye el estereotipo generacional sobre las niñas y los niños como inferiores, además de que se brinda la base ideológica de los discursos que legitiman el poder y la autoridad de los adultos (Paves, 2012).

Por lo tanto, entender los ambientes culturales en los que se producen las formas de atención a la infancia es fundamental porque "las concepciones en torno a los procesos étnicos, de género y por condición migratoria han sido definidas como 'diferencias culturales' y de minorías sociales. Ello conduce a distintas políticas de 'atención', entre ellas las educativas, visibilizando dichas diferencias y endureciendo sus parámetros de distinguibilidad" (Medina, 2007: 172).

De esta forma, el problema de la diversidad de acepciones de los conceptos y discursos que se supone responden ideológicamente al reconocimiento de la diversidad cultural, como el multiculturalismo, el pluriculturalismo o el interculturalismo, "reside en la comprensión de los marcos de referencia sociohistóricos y los espacios siempre asimétricos que condicionan dichas relaciones, las cuales no son culturales en sí mismas, sino sociopolíticas y económicas" (Medina, 2007: 174-75). Debido a ello, los antagonismos reales en las guerras, la dominación y la explotación no son la cultura, la raza o la religión, "pero la idea racista de la diferencia, justifica muy bien los hechos neoliberales de despojo, dominación y hace deseable la competencia, de tal suerte que los racismos resultan funcionales a los intereses belicistas" (Molinari y Enríquez, 2014: 208).

Por eso, las relaciones que se asumen interculturales en contextos específicos no son culturales en sí mismas, es decir, la asimetría no se establece por las diferencias de códigos comunicativos, sino por el marco de relaciones que genera discursos o referentes a esas culturas para legitimar y encubrir las relaciones de desigualdad a través de clasificaciones sociales que sirven para naturalizar la estructura de poder. 
De ahí la importancia de identificar los modelos de atención a la infancia indígena en Chiapas como parte del trabajo de investigación y vinculación que desarrolla el Cuerpo Académico "Infancia y juventud en contextos de diversidad", ${ }^{1}$ con el objetivo de sistematizar y analizar información sobre los modelos de atención que subyacen en las acciones realizadas por las instancias que atienden a esta población para establecer puentes de acción a partir de la vinculación de la academia con las organizaciones sociales.

\section{Modelos de atención a la infancia en Chiapas}

Nuestro objetivo en este punto de la investigación consistió en iniciar un acercamiento a las distintas instancias de atención a la niñez en Chiapas, identificar su núcleo de acción y elegir las más representativas con la intención de conocer sus modelos y propuestas de intervención particulares. Partimos de la premisa de que ciertas perspectivas teórico-metodológicas orientan el trabajo de estas organizaciones vinculadas con infantes indígenas, pero también concepciones de lo que es la infancia y lo indígena. En este sentido, resulta relevante definir estas perspectivas con la finalidad de conocer las concepciones que subyacen en sus modelos de atención, así como las particularidades que puedan aportar a los estudios actuales sobre la infancia y a las políticas de atención a esta población en su diversidad de contextos.

En esta región chiapaneca se han realizado trabajos de investigación acerca de las condiciones de vida de la niñez y los órganos orientados a su atención; particularmente, existen algunos estudios acerca de los niños trabajadores en las calles de San Cristóbal de Las Casas (Tovote y Maynard, 2010; Necasová y Escalona, en prensa; Melel Xojobal, 2012; Pérez, 2012)

Algunos de estos trabajos han estado dirigidos a la evaluación de los modelos y políticas de atención a la niñez, implementados por organizaciones o dependencias, entre las que destacan la asociación civil Melel Xojobal y la Red por los Derechos de la Infancia y la Adolescencia en el Sureste Mexicano. Sin embargo, aún no se ha realizado una sistematización en la que se identifiquen los diversos centros que atienden a este sector de la población en el estado y en la que también se expliciten sus concepciones acerca de la infancia, considerando los actuales estudios de la niñez, así como los marcos legales de reciente creación.

Lo anterior, porque otros proyectos de investigación en la región relativos a la infancia se han enfocado en las formas propias de participación de los niños y niñas en sus contextos culturales, y han señalado la importancia de reconocer la diversidad de infancias, la participación y la voz de los niños y las niñas en sus entornos más cercanos (Bermúdez y Núñez, 2009; De León, 2010; Gaskin, 2010; Núñez y Alba, 2013).

Nuestra investigación se dirige a identificar los diversos centros de atención a la infancia en Chiapas, y los resultados que presentamos en este artículo se centran en aquellos que contemplan la condición étnica y la educación como ejes para su acción. Lo anterior con la intención de señalar cómo las políticas de atención a la interculturalidad siguen enfocándose a sólo una de las dimensiones culturales: la étnica, en entornos donde la presencia de población indígena es importante, y se invisibilizan otras, como la clase, el género o la religión. Sin embargo, fuera de estos lugares la condición étnica se difumina, se imponen las visiones de homogeneidad cultural en la atención y, sin embargo, se mantienen las relaciones de racismo y discriminación.

Esta relación entre "etnia" y "raza" es compleja porque, de acuerdo con Gilberto Giménez (2007), la idea de "raza" como traductor de una supuesta división genética y bilógica entre los grupos humanos no es un concepto científico, frente al concepto de "etnia" como traductor de una realidad que refiere a comunidades culturales existentes, por lo que "raza" debería subsumirse bajo el concepto de etnia. Sin embargo, este argumento no aplicaría de la misma forma a la relación entre "racismo" y "etnicismo", porque:

[...] racismo es una ideología (o prejuicio o representación social) por lo que se inferioriza y discrimina a ciertas comunidades étnicas sólo por sus atributos biológicos o fenotípicos, dejando de lado los demás atributos culturales [... que] no sólo existe, sino que es la forma de discriminación que 
más duele y la que más lesiona la identidad propia y la autoestima. [...] "Etnicismo" es también una ideología que discrimina a comunidades étnicas sólo en virtud de sus diferencias culturales (Giménez, 2007: 42).

De esta manera, las relaciones de dominación que encubren estas ideologías esencializan a las culturas e imponen la homogeneidad en la atención a las diversas infancias. Lo anterior exige mayor claridad en el uso de conceptos como "cultura" e "interculturalidad", y nuevas formas de abordar la compleja trama que los implica en la construcción de las relaciones sociales, debido a que el uso indiscriminado de estos conceptos en diversas disciplinas "a menudo los reifica y esencializa hasta tal grado que se vuelven abiertamente contraproducentes tanto para el análisis académico como para el acompañamiento de procesos de transformación social o educativa" (Dietz, 201l: 5). De ahí que aún se vea lejana la educación intercultural para todos.

\section{La identificación de los centros de atención a la infancia}

La ruta metodológica se sitúa en los métodos cualitativos de investigación, y se "parte del supuesto de que todos los seres humanos, como elementos previamente socializados, somos a la vez producto y productores de un mapa mental de carácter intersubjetivo que modela nuestra percepción de la realidad" (Denman y Haro, 2000: 38). De ahí que los sujetos con los que hacemos investigación, a través de entrevistas a profundidad, no son meros informantes a los que hay que "sacar información", sino sujetos-agentes que también participan en la producción social, reflexivos de su realidad y que dan cuenta de las particularidades de los contextos en los que participan.

Para la investigación resultó importante conocer, desde los propios sujetos, las formas particulares de los modelos de atención a la infancia, de los cuales se desprenden las concepciones o imágenes que se tienen de esta población; en particular los modelos que consideraban la condición étnica.
En la primera etapa se realizó una búsqueda por internet $^{2}$ en la que se logró identificar 32 centros de atención infantil en Chiapas y se pudo reconocer su organización y su eje de acción (ver cuadro 1$){ }^{3}$

Después de la identificación se realizó una clasificación preliminar en la que se observó un número considerable de centros que clasificamos como "no identificados", los cuales se encontraron en páginas comerciales o del gobierno, pero no se logró ubicar su página web y los datos que se proporcionaban no se pudieron confirmar; por ejemplo, números telefónicos en los que no contestaban o que no existían. Lo anterior deja pendiente la importante tarea de rastrear este tipo de centros, que eventualmente pueden realizar actos de corrupción al operar como fantasmas y ser una vía para cubrir el desvío de recursos o, en el peor de los casos, violar los derechos de niñas y niños.

Este dato se suma a lo expuesto en el pasado VI Congreso Mundial por los Derechos de la Infancia y la Adolescencia, realizado en la ciudad de Puebla, México, en noviembre de 2014, en donde se destacó una constante en la falta de información que pudiera dar cuenta del número real de niñas y niños en su diversidad de condiciones y de la situación de vulnerabilidad en la que se pudieran encontrar, ya que el Estado mexicano no tiene el registro, y tampoco el control, de un sinnúmero de centros que operan sin supervisión y de los que incluso ni siquiera se tiene la certeza de que existan.

De los 32 centros de atención infantil registrados, una tercera parte se dedican explícitamente a la práctica del cuidado: hospedaje - seis-, alimentación —uno-y salud - tres- Definimos el cuidado como todo trabajo, doméstico o social, dirigido a mantener condiciones de vida sana entre la población infantil. Esta prevalencia de las instituciones de cuidado, de entrada, evidencia abandono familiar, de la comunidad y del propio Estado, que no ha garantizado educación ni salud para estos infantes. Demuestra también que hay una pauperización de las redes tradicionales de ayuda mutua que afecta la vida de niños y niñas migrantes o en situación de calle. Así, identificamos centros que abarcan varios ejes de atención, pero también están los que se enfocan a un aspecto en particular, por ejemplo, 
aquellos que atienden sólo las necesidades de niños trabajadores o de pequeños maltratados, de los que encontramos uno de cada uno. Por otro lado, apenas dos de los centros se dedican al arte y la cultura.

No obstante, el segundo eje en importancia, por ser uno de los servicios que más se ofrece, es el educativo, lo que nos lleva a cuestionar si los servicios educativos que imparte el Estado atienden las necesidades de la diversidad de existencias de la niñez; de ahí el reto de la educación intercultural que, frente a la demanda de los derechos de los pueblos originarios, se ve interpelada también por las necesidades de la niñez migrante y trabajadora (Bertely et al., 2013). Estas diversas formas de existencia infantil exigen una mayor articulación en el análisis interdisciplinario, para contribuir al abordaje de temas como la complejidad de las relaciones en contextos de diversidad y la pertinencia de los servicios educativos que atienden esta diversidad.

La clasificación preliminar de los centros de atención infantil a partir de la orientación de sus acciones (ver cuadro 2) fue un proceso complejo y no concluido porque varios de ellos trabajan diferentes ejes de forma articulada; por ejemplo, Melel Xojobal, Save the Children y Chantiik Tak Tajinkutik A.C. trabajan de manera activa con niños trabajadores, pero con un énfasis político en la promoción, defensa y ejercicio de los derechos de la niñez y la juventud indígenas, en particular el derecho al trabajo, por lo que decidimos ubicarlos en el eje de los derechos de los niños, niñas y jóvenes indígenas desde un posicionamiento políticamente explícito. En cuanto a La Casa de las Flores, se observó, a través de la información de su página web, que el énfasis se presenta en ofrecer el servicio de una casa de día, donde "pueden llegar unas horas para estudiar, jugar, descansar, comer y ser tratados con cariño", en un sentido más asistencialista y desde un posicionamiento proteccionista que considera el trabajo infantil violatorio a los derechos de la niñez.

Para los objetivos del proyecto este tipo de concepciones son fundamentales, y se podrá ahondar en ellas con entrevistas a profundidad a las dos primeras organizaciones para identificar las imágenes de infancia a partir de las cuales articulan sus modelos de atención; es decir, si se reconoce y promueve la agencia y voz de los niños y las niñas, o se mantiene la visión adultocéntrica en su acción, y si se valoran sus contextos culturales de actuación.

Por lo anterior, podríamos señalar dos posiciones encontradas: por una parte la de Melel Xojobal, que defiende los derechos de los niños y niñas, como la demanda de un trabajo digno, así como las repercusiones que esta demanda implicaría, por ejemplo, sobre el sistema educativo, el cual tendría que generar condiciones que permitieran este doble ejercicio de derechos - el del trabajo y la educación-, porque, de acuerdo con Manfred Liebel, "uno de los puntos más importantes es dejar de creer que el derecho a la educación se contrapone al derecho a trabajar" (Melel Xojobal, 2011: 237).

La otra posición, muy arraigada y que invisibiliza el protagonismo infantil, es la del discurso proteccionista desde el que, antes que el derecho a la participación, está el de la protección. No se niega la obligación social hacia la protección de la niñez, pero coincidimos con Liebel en las posibilidades de "pensar al revés, de qué manera la participación puede contribuir a la protección" (Melel Xojobal, 2011: 238).

Del total de los centros de atención a la infancia identificados, como señalábamos, un porcentaje alto - más de la tercera parte- se aboca a realizar tareas de asistencia y cuidado. Tanto las "casas de día", las "casas hogar" o las "aldeas infantiles", como las organizaciones que atienden a niños con problemas de salud, discapacidad y alimentación, se ubican en un modelo de atención asistencialista, es decir, buscan solventar las necesidades básicas de los pequeños, manteniendo con ello del lado adulto la toma de decisiones sobre los infantes.

Por otro lado están los centros que, mediante la educación y la creatividad, pretenden que niñas y niños cuenten con más herramientas para expresarse y para desenvolverse en la sociedad. Los centros de atención a la infancia que impulsan a los infantes trabajadores o en situación de calle a insertarse en la educación formal consideran que la escolarización les permitirá mejorar sus condiciones de vida - tanto en términos de 
conocimiento, como de socialización y convivencia-, e incluso sus condiciones laborales. En cuanto a la expresión artística, son pocos los órganos que centran su interés en el arte y la cultura.

En los ejes de atención presentados en la clasificación preliminar ${ }^{4}$ para identificar la acción de estos centros, encontramos que la demanda por trabajo de niñas y niños, que en su mayoría son indígenas y trabajan al lado de sus familias, exige del sistema educativo una reconfiguración que parta de sus necesidades propias y de la existencia real de la diversidad de infancias. De ahí que gran parte de la razón de ser de estos centros sea la de asumir las acciones que el Estado no atiende, como las necesidades educativas de niñas y niños trabajadores.

Por ejemplo, en Save the Children desarrollan un proyecto que pretende un nivel de integralidad en la atención a los infantes, al vincularse con las escuelas de las orillas de San Cristóbal, a las que asiste un importante número de niñas y niños trabajadores, la mayoría de ellos de origen indígena. No sólo trabajan con los pequeños y sus familias, sino que además lo hacen con los maestros y las escuelas, con la finalidad de que consideren las condiciones particulares de estos infantes:

Nuestra percepción consiste en que pasando Periférico los niños pierden su identidad de manera drástica porque, además, la misma escuela provoca que ya no hablen su lengua y no usen su indumentaria tradicional y ahora portan su uniforme [...] Pasando el Periférico incluso ocultan su identidad cultural (entrevista Save the Children, 2015).

En el caso específico de Melel Xojobal, esta organización desarrolla programas participativos de inserción y permanencia escolar para infantes trabajadores, además de buscar reducir los riesgos que existen en las calles, mediante estrategias de vinculación con los menores, con la finalidad de identificar y exponer los problemas que encuentran en su ambiente laboral. Además, promueven la participación infantil para generar conductas de autocuidado ante los riesgos que enfrentan. Sin esta atención, los niños y niñas tendrían menos posibilidades de inserción en la educación formal, ya que la escuela no reconoce su condición de trabajadores, que es, además, una demanda de la propia infancia trabajadora.
Algo de suma importancia que se analiza en Melel se relaciona con la apropiación de los derechos de la infancia. Se busca que los niños conozcan cuáles son sus derechos y obligaciones; en lo referente a sus derechos, se trabaja en cuestiones como la salud, educación, trabajo, la libre asociación y expresión y una vida libre de violencia. Es claro que no se puede cambiar la condición en que se encuentran los infantes, pero la asociación intenta otorgarles las herramientas para que tengan la capacidad de tomar las mejores decisiones (entrevista Melel Xojobal, 2015).

Además de esta condición de clase que Melel Xojobal integra en sus acciones de atención, se explicita la condición étnica, porque los niños y sus familiares con quienes trabajan son personas pobres y discriminadas por el hecho de ser indígenas. Los datos que ofrece Melel Xojobal son contundentes para dar cuenta del problema que enfrentan los niños y las niñas indígenas en San Cristóbal de Las Casas, lugar donde opera la organización. Según sus registros, 2594 niñas y niños trabajan, y 1205 acompañan a sus familias mientras éstas realizan sus actividades laborales; de estos pequeños, nueve de cada diez son indígenas.

\section{Infancias indígenas}

La construcción del niño como sujeto de estudio que pone el énfasis en sus cualidades como sujeto activo en el proceso de aprendizaje de su cultura, está abriendo alternativas de análisis bajo la premisa de que la cultura es aprendida y no interiorizada. De hecho, los niños aprenden en su cultura, considerando que el proceso de aprendizaje es diverso y depende en gran medida del interés y el contexto cultural que sustenta el tipo de relaciones que los niños establecen en él (Vigotsky, 1988; Rogoff, 1993). 
En este sentido, emergen reformulaciones dentro de las ciencias sociales y de las humanidades que exploran la agencia del niño como sujeto que aprende su cultura, y que también construye su propia cultura, en la que puede reproducir, cuestionar, negociar, transformar y crear su realidad, por lo que se da el paso necesario para pensar la infancia como un fenómeno social complejo y con múltiples dimensiones, tema que se ha comenzado a estudiar desde diversas orientaciones teóricas.

Sin duda, los estudios disciplinarios son relevantes al destacar la agencia de los niños y niñas, pero interesa destacar que es en los estudios interdisciplinarios (De León, 2010; Frisancho et al., 2011) donde se están generando ideas y formas novedosas para desarrollar la investigación sobre la niñez. Sobresalen los estudios articulados con la sociología, la antropología social y cultural, la lingüística, la educación y la psicología cultural, que han dirigido la atención al:

[...] reconocimiento del papel crítico que juega la lengua en la creación de las identidades en las culturas infantiles. Conceder a los niños su papel como agentes socializadores, reconocer sus espacios de creatividad cultural en el juego, el pleito, la discusión, la producción de historias, se vuelve central para pensar en la especificidad cultural no sólo de 'La Cultura' — siempre entendida con el parámetro 'adulto'-, sino de 'las culturas', que incluyen la diversidad cultural y lingüística en el mundo adulto e infantil (De León, 2010: 14).

En su estudio sobre niñas y niños indígenas que trabajan en San Cristóbal de Las Casas, Norma Pérez se refiere a ellos como li tsebetike sch'iuk keremetike trabajadores:

Al inicio de la redacción se utilizaban los términos niñas y niños y jóvenes, pero al reflexionar sobre el sentido y el alcance que tienen estas palabras para las familias tsotsiles, me di cuenta de que las palabras que son prestadas y que cumplen con definiciones y nociones sobre un deber ser propias de un contexto distinto, solamente sirven para forzar a las familias para pensar en español, cuando en las prácticas cotidianas estos conceptos carecen de un significado real en la experiencia cotidiana de vida en la ciudad con las familias con las que se trabajó (Pérez, 2012: 2).

Al describir la participación de los niños pequeños mayas yucatecos en su mundo, Suzanne Gaskins advierte que: "el acto de simplemente describir la actividad de los niños sin tomar en cuenta los principios culturales de la participación que definen el contexto podría conducirnos a malentendidos de consideración en la interpretación de los significados de la actividad" (2010: 40).

Por lo tanto, es crucial reconocer las diversas infancias que se expresan en contextos culturales concretos, atendiendo lo que Patricia Medina (2007) señala como producto de la historicidad de los contextos de las colectividades implicadas en la configuración de prácticas identitarias específicas.

Esto es así porque, en las actividades cotidianas de los contextos indígenas:

[...] compartir, colaborar y contribuir todos los días, no sólo llevan a la acumulación de conocimientos y habilidades por haberse familiarizado a través del tiempo con las herramientas, los materiales y los procesos relacionados con las actividades compartidas aprendidas. También dan lugar a una experiencia emocional y a una atracción hacia la actividad del contexto social en el que se llevan a cabo (Paradise, 201l: 55).

Con estas perspectivas que conceptualizan al niño como un sujeto activo en el proceso de aprendizaje en su cultura, se rechazan las premisas que sostienen que la cultura es interiorizada y aquellas en las que se considera a los niños como sujetos pasivos; por lo tanto, es fundamental conocer las formas y condiciones en que se da la participación infantil en sus contextos específicos; por ejemplo, Yolanda Corona y Carlos Pérez señalan que en la lógica cultural de los pueblos originarios se parte de una noción de pertenencia a la colectividad donde la infancia tiene una presencia activa en todos los espacios:

La noción de participación en sí misma implica que el sujeto se sienta parte de algo más amplio; en los 
pueblos indígenas se permite e incluso se espera que las nuevas generaciones cooperen, se coordinen e integren en actividades colectivas que tienen que ver con el 'bien común', aun cuando para el observador externo, éstas no se desarrollen en 'contextos seguros' para los niños (Corona y Linares, 2007: 12).

Frente al avance de los estudios teóricos, y bajo el supuesto de estar influidos por éstos, en los últimos años se han implementado en el estado de Chiapas proyectos desde distintas instancias encaminados a la atención de la niñez en alguno o algunos de los ámbitos de sus múltiples necesidades, como la educativa, la salud, o su condición de migración, en situación de calle, violencia doméstica, tráfico o explotación laboral y sexual. Frente a la diversidad de propuestas, son particularmente importantes los proyectos dirigidos a la población de niños indígenas, por ser uno de los grupos a los que más se les vulnera sus derechos.

\section{Conclusiones}

Este primer acercamiento al análisis de la infancia indígena a partir de la identificación de los centros de atención a la infancia es un comienzo que aporta a la comprensión de la diversidad de existencias de la niñez e invita a tomarse con seriedad que las niñas y los niños demandan con voz propia sus derechos, uno de ellos al trabajo digno, tema que despierta posiciones encontradas y conflictivas, pero que deja al descubierto las estructuras de poder en las que los infantes sobreviven.

Es de hacer notar que los centros de atención infantil en Chiapas responden principalmente a modelos de asistencia y cuidado, en la búsqueda de atenuar las carencias de muchos infantes que se encuentran en condiciones desfavorables. En particular destaca el trabajo de Melel Xojobal, que frente a huecos profundos en los marcos legales para la defensa de los derechos de la niñez, que denotan un problema estructural, se enfoca en dotarlos de herramientas para promover su participación en la toma de decisiones.

Sin embargo, en este reconocimiento de la participación:
[...] diversas organizaciones no gubernamentales (ONG) que se empeñan en la promoción de los derechos suelen fundamentarse en los llamados derechos de participación que establece la Convención sobre los Derechos del Niño (CDN) de Naciones Unidas. En muchos países fuera de Europa y de América del Norte, la resistencia y el escepticismo frente a estos derechos son considerables. Sus defensores son acusados de despreciar las culturas locales, sus relaciones y jerarquías generacionales, de actuar con espíritu misionero o con intenciones neocoloniales para imponer un concepto "occidental" de infancia y de los derechos del niño (Liebel y Saadi, 2012: 124).

La Convención sobre los Derechos del Niño y leyes nacionales como la Ley General de los Derechos de Niñas, Niños y Adolescentes, aprobada en México en 2014, se pueden consideran avances significativos porque reconocen como derecho la participación de la niñez; no obstante, al no establecer mecanismos que abran o generen condiciones para que realmente se dé dicha participación desde las propias formas socioculturales de las diversas infancias, se corre el riesgo de generar nuevas retóricas, tanto gubernamentales como académicas, en torno a la participación infantil que no cuestionen la universalización de derechos y las paradojas de las propuestas del multiculturalismo e interculturalidad del Estado. Esto se debe a que la participación infantil se suele limitar a los esquemas institucionales o formales de la política basada en las teorías de la democracia, que no consideran la integración de la niñez en los asuntos del quehacer político. De ahí que organizaciones como Melel Xojobal prioricen acercar las herramientas para que sean las niñas y los niños quienes tomen sus propias decisiones, es decir, para que ejerzan su participación política en lo que les es común.

En relación con la condición étnica, se pudo observar que fuera de San Cristóbal de Las Casas ésta se invisibiliza, por lo que al parecer este elemento de identidad pierde importancia, o se retoma la condición de migrante para distinguir a los niños y niñas cuando se desplazan de sus municipios o países de origen como eje para dar atención. Sin embargo, sí operan prácticas 
racistas y etnicistas que discriminan a esta población incluso desde los espacios escolares, como señalan en Save the Children, porque son las mismas escuelas las que obligan a las niñas y niños al cambio dirigido y a ocultar su identidad cultural, al promover los valores de la cultura dominante en detrimento de las culturas indígenas.

Esta situación es recurrente también en las escuelas del subsistema intercultural bilingüe, como se puede observar en el compendio del Consejo Mexicano de Investigación Educativa (COMIE) en materia de "multiculturalismo y educación" (Bertely et al., 2013), porque la lengua materna y las prácticas y saberes culturales de los pueblos y comunidades indígenas siguen siendo temas pendientes, así como la educación intercultural para todos, lo que evidencia que el discurso liberal del multiculturalismo es aún dominante en materia de políticas públicas en contextos de diversidad y está limitado a cuotas compensatorias de nivelación. Además, la interculturalidad continúa considerando sólo la condición étnica y deja fuera las demás configuraciones de diversidad, como la clase, la religión y la condición de migrante y trabajador.

Lo anterior deja pendientes tareas urgentes que comprometen a los diferentes agentes que actúan en favor de la participación real de niños y niñas para abrir los espacios en los que, de acuerdo con sus diversas condiciones, se puedan expresar en un marco de respeto y dignidad. Uno de los espacios en los que se exige no sólo una apertura, sino una reconfiguración, es la educación pública en general, que necesita interculturalizarse desde un proyecto político que transforme las relaciones de colonización aún vigentes y que integre las necesidades de esta diversidad de existencias, así como la valoración e integración explicita de prácticas comunitarias para el aprendizaje de la participación colectiva, con el aporte de los pueblos originarios que es de urgencia reconocer.

\section{Notas}

${ }^{1}$ Este proyecto vigente es financiado por el Programa para el Fortalecimiento de Cuerpos Académicos del Programa para el Desarrollo Profesional Docente para el tipo superior (PRODEP) 2014, en el que participamos las que suscribimos, además de la Mtra. Norma G. Pérez López, colaboradora del cuerpo académico, y estudiantes de las licenciaturas de Historia y Antropología Social de la Facultad de Ciencias Sociales de la Universidad Autónoma de Chiapas: Enrique A. Jiménez Gómez, Gustavo Navarro León, Fernanda Villa Saijas y Orlando Ruíz López.

2 La búsqueda se realizó en sitios que concentran información sobre infancia y juventud, siendo uno de los más importantes el de Infoniñez.mx, de la UNICEF México - http://www.infoninez.mx/—, además del sitio del DIF Chiapas - http://www.difchiapas.gob.mx/-.

${ }^{3}$ En este cuadro se señala, además, el tipo de contacto realizado, y en gris se marcan los centros que aceptaron participar en la investigación.

${ }^{4}$ Esta clasificación fue realizada con base en la información recabadaen páginas de internet, se corroboró telefónicamente y se realizó la selección de los centros. Para elaborar el cuadro incluimos sólo ciertas instituciones. Se descartaron las que no identificaban comoejecentral de sutrabajolacondiciónétnica de los infantes. En cuanto a aquellas en las que no quedaba explícito su eje de atención, se estableció comunicación telefónica, pero las que no señalaron el eje étnico también fueron descartadas. Finalmente identificamos aquellas que tenían como eje central la condición étnica y se logró el enlace personal, y se consiguió que siete organizaciones participaran y se realizaran las entrevistas a profundidad (ver cuadro 1). Queda pendiente el análisis de dichas entrevistas, ya transcritas, que aportarán mayor información para la comprensión de las diversas concepciones de la infancia que subyacen en la práctica de las instituciones de atención a este sector de la población.

\section{Referencias bibliográficas}

Bermúdez Urbina, Flor Marina y Kathia Núñez Patiño (2009). Socialización y aprendizaje infantil. Un contexto intercultural. Chiapas, México: UNICAH.

Bertely Busquets, María et al. (coord.) (2013). Multiculturalismoyeducación. 2002-2011. México: ANUIES. Corona Caraveo, Yolanda y María Eugenia Linares Pontón (2007). "Promoviendo la participación infantil y juvenil en el marco de la construcción de ciudadanía”. En Yolanda 
Corona y María Eugenia Linares, Participación infantil y juvenil en América Latina. México: UAM, Childwatch International Research Network y Universitat de Valencia. Disponible en http://www.uam.mx/cdi/ partinfantil/capl.pdf (consultado el 10 de octubre de 2010).

De León Pasquel, Lourdes (coord.) (2010). Socialización, lenguajes y culturas infantiles: estudios interdisciplinarios. México: CIESAS.

Denman, Catalina A. y J.A. Haro (comp.) (2000). Por los rincones. Antología de métodos cualitativos en la investigación social. Hermosillo, Sonora: El Colegio de Sonora.

Dietz, Gunther (2011). "Hacia una etnografía doblemente reflexiva: una propuesta desde La antropología de la interculturalidad". En Revista de Antropología Iberoamericana, vol. 6, núm. 1, enero-abril, pp. 3-26.

Frisancho, Susana et al. (ed.) (2011). Aprendizaje, cultura y desarrollo. Una aproximación interdisciplinaria. Perú: Fondo Editorial de la Pontificia Universidad Católica del Perú.

Gaitán Muñoz, Lourdes (2006). "La nueva sociología de la infancia. Aportaciones de una mirada distinta". En Políticay Sociedad, vol. 43, núm. 1, pp. 9-26.

Gaskins, Suzanne (2010). "La vida cotidiana de los niños en un pueblo maya: un estudio monográfico de los roles y actividades construidos culturalmente". En Lourdes de León Pasquel (coord.), Socialización, lenguajes y culturas infantiles: estudios interdisciplinarios. México: CIESAS, pp. 37-76.

Giménez, Gilberto (2007). "Formas de discriminación en el marco de la lucha por el reconocimiento social". En Olivia Gall (coord.), Racismo, mestizaje y modernidad: visiones desde latitudes diversas. México: UNAM, pp. 37-61.

Hirschfeld, Lawrence A. (2002). "Why Don't Anthropologists Like Children?" En American Anthropologist, vol. 104, núm. 2, junio, pp. 611-627.

James, Allison, C. Jenksy A. Prout (1998). TheorizingChildhood. Nueva York: Teachers College Columbia University.

Liebel, M. e I. Saadi (2012). "La participación infantil ante el desafío de la diversidad cultural". En Desacatos, Revista de Antropología Social, núm. 39, mayo-agosto, pp. 123-140.

Medina Melgarejo, Patricia (2007). "Configuración de fronteras, interculturalidad y políticas de identidad". En Tramas, núm. 28, México, pp. 171-194.
Melel Xojobal A.C. (2011). "Derechos de la infancia, autonomía y procesos organizativos: entrevista al Dr. Manfred Liebel". En Rayuela, núm. 2, pp. 233-238.

Melel Xojobal A.C. (2012). Infancia trabajadora en San Cristóbal de Las Casas, Chiapas. Transformaciones y perspectivas a 10 años (2000-2010). San Cristóbal de Las Casas, Chiapas: Melel Xojobal.

Molinari, Claudia y Mariana Enríquez (2014). "Antropología de la orilla y Cuba transamericana: los aportes de Fernando Ortiz al pensamiento en América Latina". En Frontera Norte, vol. 26, núm. 52, diciembre, pp. 205-213.

Necasová, Lucie y José Luis Escalona (en prensa) "Educación en derechos humanos en Chiapas, México. Infancia trabajadora en Melel Xojobal".

Núñez Patiño, Kathia y Cecilia Alba Villalobos (2013). "Educación intercultural y escuela zapatista". En Gabriel Ascencio Franco (coord.), Teoría y práctica de la educación intercultural en Chiapas. Chiapas, México: PROIMSE-IIA-UNAM, pp. 91-102.

Paradise, Ruth (2011). “¿Cómo educan los indígenas a sus hijos? El cómo y el porqué del aprendizaje en la familia y la comunidad". En Susana Frisancho et al. (ed.), Aprendizaje, cultura y desarrollo. Una aproximación interdisciplinaria. Lima: Fondo Editorial de la Pontificia Universidad Católica del Perú, pp. 41- 58.

Pavez Soto, Iskra (2012). "Sociología de la infancia: las niñas y los niños como actores sociales". En Revista de Sociología, núm. 27, pp. 81-102.

Pérez López, Norma G. (2012). Socialización y trabajo desde la perspectiva de li tsebetike xch'iuk keremetike (niñas y niños) trabajadores. Tesis de Maestría en Antropología, CIESAS Unidad Sureste, México.

Rogoff, Barbara (1993). Aprendices del pensamiento. El desarrollo cognitivo en el contexto social. Barcelona: Paidós.

Tovote E. Katrin y Ashley E. Maynard (2010). “Los niños de la calle de San Cristóbal: su trabajo, su desarrollo y su futuro". En Anuario de Estudios Indígenas XIV. Chiapas, México: Instituto de Estudios IndígenasUNACH, pp. 69-98.

Vigotsky, Lev S. (1988). Pensamiento y lenguaje. México: Ediciones Quinto Sol. 


\section{Cuadro 1. Modelos de atención a la infancia indígena. Identificación vía web de los centros de atención a la niñez indígena en Chiapas*}

\begin{tabular}{|c|c|c|}
\hline Organización & Eje de atención & Tipo de contacto \\
\hline Melel Xojobal & $\begin{array}{l}\text { Es una organización social que trabaja con } \\
\text { niñas, niños y jóvenes indígenas de Chiapas, } \\
\text { en la promoción, defensa y ejercicio de sus } \\
\text { derechos a través de procesos participativos. Es } \\
\text { una de las organizaciones con trayectoria más } \\
\text { amplia de trabajo con niñas y niños indígenas } \\
\text { en el estado de Chiapas. }\end{array}$ & $\begin{array}{l}\text { San Cristóbal de Las Casas. } \\
\text { Accedieron a participar. }\end{array}$ \\
\hline Vientos Culturales & $\begin{array}{l}\text { Ayudan a desarrollar la creatividad, habilidades } \\
\text { e inteligencia, a aumentar su autoestima y } \\
\text { la seguridad, y a promover valores como } \\
\text { la honestidad, el respeto, el trabajo, el } \\
\text { estudio y el servicio. Su principal vía es el } \\
\text { arte y trabajan con los niños y jóvenes más } \\
\text { necesitados. }\end{array}$ & $\begin{array}{l}\text { Tuxtla Gutiérrez. } \\
\text { Hubo contacto telefónico pero, de } \\
\text { acuerdo con la información recopilada, } \\
\text { no hay niños hablantes de lenguas } \\
\text { originarias en su grupo de trabajo, } \\
\text { aunque en su página indican que trabajan } \\
\text { en comunidades indígenas. }\end{array}$ \\
\hline Manos Amigas & $\begin{array}{l}\text { Es una Asociación Civil sin fines de lucro, } \\
\text { formada por un grupo de personas de diferente } \\
\text { condición y profesión, que trabajan unidas para } \\
\text { aportar lo que nos sea posible, para socorrer } \\
\text { a la niñez y adolescentes más necesitados } \\
\text { de las comunidades, pueblos y ciudades de } \\
\text { nuestro estado de Chiapas, y al mismo tiempo } \\
\text { ir construyendo una convivencia social más } \\
\text { humana y participativa entre nosotros. }\end{array}$ & $\begin{array}{l}\text { Tuxtla Gutiérrez y San Cristóbal de Las } \\
\text { Casas. } \\
\text { No se logró hacer el contacto. }\end{array}$ \\
\hline Suekun Sueniños A.C. & $\begin{array}{l}\text { Trabajan con niñas y niños indígenas, y con } \\
\text { niños marginados, a través de una educación } \\
\text { complementaria a la escuela. }\end{array}$ & $\begin{array}{l}\text { San Cristóbal de Las Casas. } \\
\text { Accedieron a participar. }\end{array}$ \\
\hline La Casa de las Flores & $\begin{array}{l}\text { Trabajan con niñas y niños indígenas, sobre } \\
\text { todo aquellos que trabajan en el centro de la } \\
\text { ciudad, pero lo hacen a "puerta cerrada". Es } \\
\text { una casa de día y pueden llegar unas horas para } \\
\text { estudiar, jugar, descansar, comer y ser tratados } \\
\text { con cariño. }\end{array}$ & $\begin{array}{l}\text { San Cristóbal de Las Casas. } \\
\text { Si hubo contacto pero no participaron. }\end{array}$ \\
\hline $\begin{array}{l}\text { Hogar Funcional } \\
\text { para Niñas y Niños } \\
\text { Mensajeros de la Paz } \\
\text { Chiapas A.C }\end{array}$ & $\begin{array}{l}\text { Educación y protección a los derechos de la } \\
\text { niñez. Atienden únicamente a un niño hablante } \\
\text { de tsotsil. }\end{array}$ & $\begin{array}{l}\text { San Cristóbal de Las Casas. } \\
\text { Hubo contacto telefónico con la } \\
\text { coordinadora, pero no se concretó su } \\
\text { participación por razones de tiempo. }\end{array}$ \\
\hline Save The Children & $\begin{array}{l}\text { Trabajan con niñas y niños indígenas. } \\
\text { Su eje de trabajo consiste en inspirar avances } \\
\text { en la forma en la que el mundo trata a las niñas, } \\
\text { niños y adolescentes, y en lograr un cambio } \\
\text { inmediato y duradero en sus vidas. }\end{array}$ & $\begin{array}{l}\text { San Cristóbal de Las Casas. } \\
\text { Accedieron a participar. }\end{array}$ \\
\hline
\end{tabular}




\section{Chantiik Tak Tajinkutic A.C.}

Skolta'el Yu'un Jlumaltic A.C. (SYJAC)

Soñar Despierto A.C.

Germinalia. A.C.

\section{Albergue Infantil Salesiano, A.C.}

Albergue para Niños, San Martín De

Porres, A.C.

\section{Asociación}

Protectora Infantil

Maranatha, A.C.
Es una organización que trabaja con niños y jóvenes indígenas trabajadores. Su eje de acción consiste en contribuir a mejorar la calidad de vida digna de niños, niñas y jóvenes por medio de la formación en derechos y valores, coadyuvar a crear un ambiente social más inclusivo y desarrollar proyectos educativos y productivos

Es una organización civil. Su centro de atención son las mujeres indígenas. Su eje consiste en acompañar, fortalecer y difundir los derechos económicos, sociales, culturales, ambientales e indígenas, a través de la generación de procesos integrales en las comunidades, grupos e instituciones.

No trabajan con ningún niño hablante de lenguas originarias.

Su eje de acción consiste en generar cambios positivos tanto en la niñez desprotegida, como en la juventud, fomentando la vivencia de valores en acciones concretas como fundamento para mejorar sus vidas de manera integral.

Es una asociación juvenil dedicada a la construcción de espacios de posibilidad que transformen problemáticas concretas de las artes, medioambiente, ciencia y tecnología, educación y desarrollo social. Promueve y desarrolla acciones participativas y creativas orientadas a encontrar soluciones efectivas a problemas que enfrenta la población de contextos socialmente desfavorecidos en México, en particular, niños y jóvenes. Ofrece servicios de apoyo a grupos e instituciones en temas de educación y promoción de la cultura.

Casa Hogar. Contacto con el DIF para el servicio de adopción. Atienden a niños zoques y tsotsiles.

Casa Hogar. Contacto con el DIF para el servicio de adopción.

No hay información.
San Cristóbal de Las Casas.

Accedieron a participar.
San Cristóbal de Las Casas. Se estableció contacto personal pero no accedieron a participar.

Tuxtla Gutiérrez.

Se hizo contacto, pero no participaron por no identificar la condición étnica como eje de trabajo.

San Cristóbal de Las Casas.

No tiene como eje central la condición étnica, aunque sí consideran el contexto cultural.
Tuxtla Gutiérrez.

Accedieron a participar.

Comitán.

Se hizo contacto telefónico, pero no participaron por no identificar la condición étnica como eje de trabajo.

Tapachula.

No se encontró el contacto. Ubicada en este vínculo: http://www.tuugo.com.mx/ Companies/asociacion-protectora-infantilmaranatha/0200003731887 (consultado el 12 de febrero de 2015). 
Hogar Infantil para

Huérfanos y

Abandonados, A.C.

Centro de Creación

Artística

Infantil “Creartín”

Niños de Nueva

Independencia, A.C.

Patronato Pro Albergue Infantil Esperanza, A.C.

Patronato Refugio del Niño, A.C.

Programa 1337 de Ayuda Familiar Niños de Ciudad Cuauhtémoc, A.C.

ONG Todo por Ellos, A.C.

Casa de la Amistad

Nuestro Hogar en Chiapas, A.C.
Casa Hogar

Su eje de trabajo es el arte y la cultura.

Información encontrada en el reporte de donatarias autorizadas en 2009, en la página de la Secretaría de Hacienda y Crédito Público.

Orfanato y otras residencias de asistencia social del sector privado.

Información encontrada en la página del Diario Oficial de la Federación.

Información encontrada en el reporte de donatarias autorizadas en 2009, en la página de la Secretaría de Hacienda y Crédito Público.

Ofrece refugio nocturno, cena y desayuno a niños, niñas y adolescentes en situación de calle.

Atención a niños con cáncer. Proporciona medicamentos.

Es un centro que tiene como eje trabajar en la recuperación para niños con VIH-Sida y sus familiares.
Ocozocoautla de Espinosa.

Se hizo contacto telefónico, pero no participaron por no identificar la condición étnica como eje de trabajo.

Tuxtla Gutiérrez.

Se hizo contacto telefónico, pero no participaron por no identificar la condición étnica como eje de trabajo.

Frontera Comalapa.

No se encontró el contacto. Ubicada en este vínculo:

http://www.shcp.gob.mx/INGRESOS/

Reporte Donatarias/reporte de donatarias_2009.pdf (consultadōol 12 de febrero de 2015).

Tapachula.

No se encontró el contacto. Ubicada en este vínculo: http://www.mxempresas. com/e/empresa/patronato-pro-albergueinfantil-esperanza-a-c/519111 (consultado el 12 de febrero de 2015).

Tapachula.

No se logró contacto. Ubicada en este vínculo:

http://dof.gob.mx/nota_detalle_popup. php?codigo $=764330$ (consultado el 15 de febrero de 2015).

Ciudad Cuauhtémoc.

http://www.shcp.gob.mx/INGRESOS/

Reporte Donatarias/reporte de donatarias_2009.pdf (consultadōo el 12 de febrero de 2015).

Tapachula.

No se encontró el contacto. Ubicación por el vínculo: http://archivos.

diputados.gob.mx/Comisiones LXII/

Participacion/04.pdf (consultad̄o el 12 de febrero de 2015).

Tuxtla Gutiérrez, San Cristóbal de Las Casas y Tapachula.

No tiene como eje central la condición étnica.

Tuxtla Gutiérrez.

No tiene como eje central la condición étnica. 


\section{El Ingenio}

Casa Taller de Artes y Oficios

Casas de Atención Infantil (CAI)

Odres Nuevos Chiapas: Casa Hogar Alegre y Dormitorio El Rancho

Aldeas Infantiles SOS Comitán

Aldeas Infantiles SOS Tuxtla Gutiérrez

Sanando Heridas, A.C.

Centro de Atención Integral Comunitario (CIAC). DIF Municipal

Comedor Solidario Manos Amigas
Trabaja con niñas y niños, jóvenes, padres y maestros de Los Altos de Chiapas, sin considerar origen étnico para sus metas organizativas.

Es una comunidad de aprendizaje desarrollada por Germinalia, A.C., que busca detonar procesos de aprendizaje y creatividad. Señalan que los niños que atienden ya no hablan una lengua nativa.

Atiende a niños de la calle. Proporciona alimentación, apoyo escolar y se les enseña un oficio a niños de 7 a 17 años. Lo denominan como un espacio abierto para niños de la calle. Es un programa del DIF municipal.

Atienden a hijas e hijos de madres y familias trabajadoras de bajos recursos. Ofrece el servicio de guardería, atención pedagógica y nutricional en las etapas inicial y preescolar. Programa del DIF municipal.

No hay presencia de niños hablantes de lenguas nativas.

Ayudan a niños abandonados y maltratados.

Es una institución de asistencia privada, independiente, no gubernamental y sin inclinación religiosa.

Atienden a niños en situación de orfandad o que han perdido el cuidado de sus padres, con un enfoque familiar en el cuidado a largo plazo.

Atiende a niños con discapacidad en Tuxtla. Nuevo proyecto de la organización.

Su misión consiste en mejorar la calidad de vida de los habitantes y comunidades en desventaja de la región de Los Altos de Chiapas, mediante atención médica y educación en salud, respetando sus usos y costumbres.

Intenta incorporar a los infantes a trabajos más dignos; busca que ya no trabajen en calles y que tengan un trabajo digno.

Alimentación. Comedor comunitario.
San Cristóbal de Las Casas.

Se hizo contacto telefónico, pero no participaron por no identificar la condición étnica como eje de trabajo.

Tuxtla Gutiérrez.

Se hizo contacto telefónico, pero no participaron por no identificar la condición étnica como eje de trabajo.

Tuxtla Gutiérrez.

No consideran la condición étnica.

Tuxtla Gutiérrez y Las Margaritas. No consideran la condición étnica.

Comitán.

No consideran la condición étnica.

Tuxtla Gutiérrez.

No consideran la condición étnica.

San Cristóbal de Las Casas.

La coordinadora mostró disposición para conversar, pero tenía tiempo limitado. No se pudo concretar la cita.

San Cristóbal de Las Casas. Accedieron a participar.

Tuxtla Gutiérrez. Accedieron a participar.

Fuente: elaboración propia.

* Se muestran señaladas en gris las organizaciones que aceptaron participar. 


\section{Cuadro 2. Clasificación de los centros de atención a la infancia en Chiapas}

\section{Tipo de orientación}

Derechos de los niños, niñas y

jóvenes indígenas

Educativas

Casa hogar

Niños trabajadores

Salud

Arte y cultura

Voluntariado

Discapacidad

Asistencia social (DIF)

Alimentación

No identificados. No se encontró el contacto
Centros de atención

Número

Melel Xojobal; Save the Children; Chantiik Tak Tajinkutik, A.C.

Sueniños; Hogar Funcional para Niñas y nNños Mensajeros de la Paz Chiapas A.C.; Germinalia, A.C.; El Ingenio; Skolta'el Yu'un Jlumaltic A.C. (SYJAC).

Manos Amigas; Albergue Infantil Salesiano, A.C.; Albergue para Niños San Martín de Porres, A.C; Hogar Infantil para Huérfanos y Abandonados, A.C.; Odres Nuevos Chiapas: Casa Hogar Alegre y Dormitorio El Rancho; Aldeas Infantiles SOS.

La Casa de las Flores.

Casa de la Amistad; Nuestro Hogar en Chiapas, A.C.; Sanando Heridas, A.C.

Vientos Culturales; Centro de Creación Artística Infantil Creartín.

Soñar Despierto A.C.

Aldeas Infantiles SOS. Tuxtla Gutiérrez

Casa Taller de Artes y Oficios (niños de la calle); Casas de Atención Infantil CAI; Centro de Atención Integral Comunitario (CIAC). DIF Municipal San Cristóbal

Manos Amigas, comedor solidario

Asociación Protectora Infantil Maranatha, A.C., Niños de Nueva Independencia, A.C., Patronato Pro Albergue Infantil Esperanza, A.C., Patronato Refugio del Niño, A.C., Programa 1337 de Ayuda Familiar Niños de Ciudad Cuauhtémoc, A.C., ONG Todo por Ellos, A.C.

Total

Fuente: elaboración propia. 